\title{
Diagnostic value of adenosine deaminase in nontuberculous lymphocytic pleural effusions
}

\author{
D. Jiménez Castro*, G. Díaz Nuevo", E. Pérez-Rodríguez", R.W. Light
}

\begin{abstract}
Diagnostic value of adenosine deaminase in nontuberculous lymphocytic pleural effusions. D. Jiménez Castro, G. Díaz Nuevo, E. Pérez-Rodríguez, R. W. Light. (C)ERS Journals Ltd 2003.

ABSTRACT: Adenosine deaminase (ADA) can aid in the diagnosis of tuberculous pleural effusions, but false-positive findings from lymphocytic effusions have been reported. The purpose of this study is to assess the ADA levels in nontuberculous lymphocytic pleural effusions (lymphocyte count $>\mathbf{5 0} \%$ ) of different aetiologies.

Altogether, 410 nontuberculous lymphocytic pleural fluid samples were consecutively selected. These included malignant effusions $(n=221)$, idiopathic effusions $(n=76)$, parapneumonic effusions $(n=35)$, postcoronary artery bypass graft surgery effusions $(n=6)$, miscellaneous exudative effusions $(n=21)$ and transudative effusions $(n=51)$.

The ADA level reached the diagnostic cut-off for tuberculosis $\left(40 \mathrm{U} \cdot \mathbf{L}^{-1}\right)$ in seven of the 410 cases $(1.71 \%)$. The negative predictive value of ADA for the diagnosis of pleural tuberculosis was $99 \%$ ( 403 of 407 cases) in the group of lymphocytic pleural effusions. In five of these seven patients $\mathrm{ADA}_{1}$ and $\mathrm{ADA}_{2}$ were measured, and in all these cases $(100 \%) \mathrm{ADA}_{1} / \mathrm{ADA}_{\mathrm{p}}$ correctly classified these lymphocytic effusions as nontuberculous $($ ratio $<0.42$ ).

This prospective study provides additional evidence that adenosine deaminase levels in nontuberculous lymphocytic pleural effusions seldom exceed the cut-off set for tuberculous effusions. The pleural fluid adenosine deaminase levels were significantly higher in different types of exudative effusions than in transudates. An adenosine deaminase level $<\mathbf{4 0} \mathrm{IU} \cdot \mathrm{L}^{-1}$ virtually excluded a diagnosis of tuberculosis in lymphocytic pleural effusions. Adenosine deaminase /adenosine deaminase $_{\mathrm{p}}$ correctly classified all nontuberculous lymphocytic pleural effusions with high adenosine deaminase levels.
\end{abstract}

Eur Respir J 2003; 21: 220-224.

\author{
*Emergency Dept and "Respiratory \\ Dept, Ramón y Cajal Hospital, \\ Madrid, Spain. Pulmonary Division, \\ Saint Thomas Hospital and Vanderbilt \\ University, Nashville, TN, USA.
}

Correspondence: D. Jiménez Castro Emergency Dept

Ramón y Cajal Hospital

28034 Madrid

Spain

Fax: 34949227502

E-mail: djc_69_98@yahoo.com

Keywords: Adenosine deaminase pleural effusion

tuberculosis

Received: June 192002

Accepted after revision: September 23 2002
The diagnostic problem of tuberculous pleuritis (TP) is emphasised as tuberculosis is a frequent pathology and due to the difficulties of its diagnostic confirmation. The pleural fluid of TP is usually predominantly lymphocytic. In fact, pleural fluid lymphocyte percentages $>85 \%$ are very suggestive of the diagnosis [1]. In one series of 49 patients with TP only five $(10 \%)$ had $<50 \%$ lymphocytes in pleural fluid [2]. Only in acute TP can an increase in neutrophils be found [3]. However, if serial thoracenteses are performed, the differential white blood cell count will reveal a change to predominantly small lymphocytes. Other causes of lymphocytic pleural effusions include malignancies, collagen vascular disease, chylothorax and post-coronary artery bypass graft (CABG) pleural effusion [4].

Pleural fluid adenosine deaminase (ADA) has been shown to be a useful biochemical marker of TP and provides a reliable basis for a treatment decision, particularly in areas where the disease is prevalent. However, the elevation may be limited in early stages of disease, and in addition, high levels of ADA can also be found in patients with neutrophilic effusions such as parapneumonic effusions or empyemas [5]
It has been shown recently that ADA levels in nontuberculous lymphocytic pleural effusions seldom exceed the cut-off set for tuberculous effusions [6]. The purpose of this prospective study is to further assess the ADA levels in a larger series of nontuberculous lymphocytic pleural effusions.

\section{Materials and methods}

All patients included in this study signed an informed consent that had been approved by the institutional review board. Lymphocytic effusions were defined as effusions with a lymphocyte count $>50 \%$ of the total nucleated cells, as conventionally defined [7].

Altogether, 410 pleural fluid samples were consecutively selected from all nontuberculous lymphocytic pleural fluids collected from patients who underwent thoracentesis at Ramón y Cajal Hospital between December 11992 and May 1 2000. Diagnostic confirmation in tuberculous pleural effusions was obtained through the identification of mycobacteria in pleural 
Table 1.-Aetiologies of lymphocytic pleural effusions

\begin{tabular}{lc}
\hline Diagnoses & Patients n \\
\hline Malignant pleural effusions & 221 \\
Lung & 138 \\
Lymphoma & 7 \\
Mesothelioma & 10 \\
Others & 66 \\
Idiopathic & 76 \\
Parapneumonic & 35 \\
Post-CABG & 6 \\
Miscellaneous exudates & 21 \\
Trauma & 7 \\
Rheumatoid arthritis & 5 \\
Chylothorax & 3 \\
Post-transplantation & 2 \\
Pancreatitis & 2 \\
Pulmonary embolism & 2 \\
Transudates & 51 \\
Congestive heart failure & 36 \\
Hepatic cirrhosis & 9 \\
Renal failure & 6 \\
Tuberculosis & 76 \\
\hline
\end{tabular}

CABG: coronary artery bypass grafting.

fluid and/or biopsy or by the presence of necrotising granulomas.

The diagnoses of the pleural fluid samples are listed in table 1. All patients underwent a thorough and uniform diagnostic work-up by two independent investigators. The definitions for the diagnosis of the effusions have been previously published [8]. A post$\mathrm{CABG}$ effusion was one that developed within the first 3 months after coronary artery bypass surgery with or without heart valve replacement, with no other identifiable causes (e.g. congestive heart failure, chylothorax or infection). A pleural effusion was categorised as malignant if pleural fluid cytology or pleural biopsy findings were positive for malignancy or if the patient had known metastatic malignancy with no other explanation for the effusion. A parapneumonic effusion was one that developed in a patient with fever, pulmonary infiltrates and complete response to antibiotic treatment. All other exudative effusions were included in the miscellaneous exudate group. An idiopathic pleural effusion was identified as one for which a cause was not determined despite an initial workup that included repeated thoracenteses and pleural biopsy. In six patients $(7.9 \%)$, the effusion resolved spontaneously during the initial work-up. The remaining 70 patients were followed up until resolution for a mean of 26 months (range 10-72, median 21) and no patient has developed tuberculosis.

The routine study of the pleural fluid included the following: $\mathrm{pH}$, biochemical testing of pleura/serum (proteins, lactate dehydrogenase (LDH), glucose, cholesterol, triglycerides, albumin and ADA), haemogram, cytology and microbiological testing (Gram, Ziehl, aerobic and anaerobic cultures, and a mycobacterial culture). The reasons for performing closed pleural biopsy were as follows: suspected malignant pleural effusions, suspected granulomatous diseases (tuberculosis, connective disorders and others) and unexplained exudate. When the diagnosis was uncertain after thoracentesis or closed pleural biopsy, the effusion persisted and the symptoms increased or malignancy was still suspected, the patient was referred for thoracoscopy and/or thoracotomy. A summary of the procedures used in the diagnostic work-up is shown in table 2.

Total ADA was determined by the Blake-Berman method [9], which had the same diagnostic properties as the Giusti-Galanti method in a meta-analysis of 2,251 cases [10]. $\mathrm{ADA}_{1}$, one of the two isoenzymes that account for the pleural activity of ADA, was determined by the Carilaos-Gakis method. In this study, the laboratory cut-off of ADA for tuberculous pleural effusion was $>40 \mathrm{IU} \cdot \mathrm{L}^{-1}$ [8] and the cut-off for $\mathrm{ADA}_{1} / \mathrm{ADA}_{\mathrm{p}}$ was $<0.42$ [8].

\section{Statistical analysis}

Results are expressed as mean \pm SD unless otherwise stated. The statistical analyses applied included the Chi-squared test with Fisher's or Yates correction, to analyse the dependence between qualitative variables, the nonparametric Mann-Whitney U-test, for continuous variables with non-normal distribution and unpaired t-tests for those with normal distribution. Distributions were considered normal or non-normal as defined by the Shapiro-Wilks test.

\section{Results}

The ADA levels in the malignant effusions (15.57 $\pm 10.60)$, parapneumonic effusions $(15.89 \pm 10.62)$, post-CABG effusions $(15.17 \pm 10.54)$, miscellaneous

Table 2. - Diagnostic work-up data for patients with lymphocytic effusions

\begin{tabular}{|c|c|c|c|c|c|}
\hline & $\begin{array}{l}\text { Closed pleural } \\
\text { biopsy }\end{array}$ & Chest CT & $\begin{array}{c}\text { Fibreoptic } \\
\text { bronchoscopy }\end{array}$ & Lung scintigraphy & $\begin{array}{l}\text { Thoracoscopy/ } \\
\text { thoracotomy }\end{array}$ \\
\hline Malignant & 207 & 106 & 83 & 15 & 3 \\
\hline Idiopathic & 71 & 60 & 8 & 15 & 5 \\
\hline Parapneumonic & 2 & 7 & 6 & 1 & 0 \\
\hline Post-CABG & 1 & 0 & 0 & 0 & 1 \\
\hline Miscellaneous & 7 & 10 & 5 & 2 & 0 \\
\hline Transudates & 3 & 0 & 0 & 1 & 0 \\
\hline Tuberculosis & 69 & 8 & 6 & 0 & 1 \\
\hline
\end{tabular}

Data are presented as patients n. CT: computed tomography; CABG: coronary artery bypass grafting. 


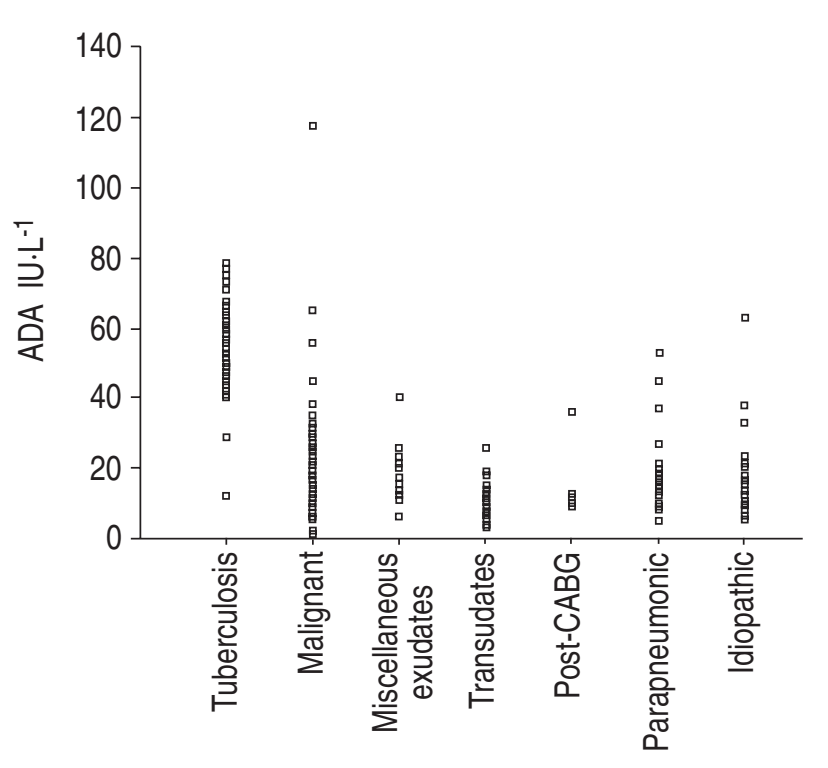

Fig. 1.-Adenosine deaminase (ADA) levels in different diagnostic groups. $\mathrm{CABG}$ : coronary artery bypass grafting.

exudative effusions (16.84 \pm 7.58$)$ and idiopathic effusions $(13.57 \pm 8.59)$ were all significantly higher when compared with the transudative group (9.55 \pm 4.50 , $\mathrm{p}<0.01$, fig. 1). The ADA level reached the diagnostic cut-off for tuberculosis $\left(40 \mathrm{IU} \cdot \mathrm{L}^{-1}\right)$ in seven of the 410 cases $(1.71 \%$, fig. 1$)$. Two patients had bronchogenic carcinomas, two had complicated parapneumonic effusions, one had a diagnosis of lymphoma, one had a mesothelioma and one case was idiopathic. In five of these patients $\mathrm{ADA}_{1}$ and $\mathrm{ADA}_{2}$ were measured and in all these cases $(100 \%) \mathrm{ADA}_{1} / \mathrm{ADA}_{\mathrm{p}}$ correctly classified these lymphocytic effusions as nontuberculous (ratio $<0.42$ ).

In this series, an ADA level $<40 \mathrm{IU} \cdot \mathrm{L}^{-1}$ excluded tuberculosis in lymphocytic pleural effusions in $99 \%$ (403 of 407) of cases.

Table 3 shows the haematological and biochemical analyses of the pleural fluid samples. There was no strong correlation between the ADA levels and the various haematological and biochemical parameters (data not shown).

\section{Discussion}

The diagnostic utility of ADA in lymphocytic pleural effusions has been evaluated. This prospective study provides additional evidence that ADA levels in nontuberculous lymphocytic pleural effusions seldom exceed the cut-off set for tuberculous effusions. Low ADA levels in lymphocytic pleural effusions virtually exclude the diagnosis of tuberculosis. The pleural fluid ADA levels were significantly higher in different types of exudative effusions than in transudates. $\mathrm{ADA}_{1} /$ $\mathrm{ADA}_{\mathrm{p}}$ correctly classified all nontuberculous lymphocytic pleural effusions with high ADA levels.

The diagnosis of tuberculous pleural effusions can be difficult because of the low sensitivity of the various diagnostic tools. A lymphocytic exudate which is seen with tuberculous pleuritis, can also occur with other diseases such as malignancy and collagen vascular diseases.

Regarding the sensitivity of diagnostic methods for tuberculous pleurisy, the positive rate with smear testing for tubercle bacilli in pleural fluid is $11.1 \%$ in the present authors' experience [8], with culturing $33.3 \%$ and with closed pleural biopsy $96.2 \%$. However, other groups have reported lower diagnostic rates for tuberculous pleural effusions $[11,12]$.

Pleural fluid ADA has long been used as a marker for tuberculous pleurisy. Levels of ADA in pleural fluid $>40 \mathrm{IU} \cdot \mathrm{L}^{-1}$ can indicate pleural tuberculosis with sensitivity $(81-100 \%)$ and specificity $(83-100 \%)$ [13-15]. The false-positive cases in the literature are mainly due to empyemas, lymphomas, malignant diseases and other aetiologies, such as parapneumonic or collagen vascular disease $[14,16]$. Examination of those studies reveals that pleural fluids of any cell type predominance were included. As in this study, previous reports $[17,18]$ have found increased ADA levels in patients with complicated parapneumonic effusions, wherein the immune response involves polymorphonuclear cells and macrophages rather than lymphocytes.

In this study, there were no empyemas in the parapneumonic effusion group as empyemas are predominantly neutrophilic effusions. This could explain the low average ADA values found in the group of lymphocytic parapneumonic effusions.

Table 3. - Haematological and biochemical analysis of pleural fluids in diagnoses subgroups

\begin{tabular}{lcccccc}
\hline Variable & Malignant & Other exudates & Idiopathic & Post-CABG & Parapneumonic & Transudates \\
\hline $\mathrm{pH}$ & $7.35 \pm 0.09^{*}$ & $7.38 \pm 0.04$ & $7.37 \pm 0.05^{*}$ & $7.40 \pm 0.02$ & $7.36 \pm 0.07^{*}$ & $7.40 \pm 0.07$ \\
$\mathrm{Glucose} \mathrm{mg} \cdot \mathrm{dL}^{-1}$ & $112.1 \pm 44.5^{*}$ & $111.4 \pm 36.8^{*}$ & $120.6 \pm 33.9^{*}$ & $168.7 \pm 78.8$ & $121.3 \pm 57.7$ & $139.4 \pm 57.6$ \\
Protein $\mathrm{mg} \cdot \mathrm{dL}^{-1}$ & $4.4 \pm 1.0^{*}$ & $5.3 \pm 4.2^{*}$ & $4.0 \pm 1.0^{*}$ & $4.1 \pm 0.8^{*}$ & $4.4 \pm 1.1^{*}$ & $2.4 \pm 1.6$ \\
Cholesterol mg $\mathrm{dL}^{-1}$ & $84.8 \pm 29.7^{*}$ & $84.6 \pm 29.1^{*}$ & $79.0 \pm 32.3^{*}$ & $80.0 \pm 27.6^{*}$ & $93.5 \pm 42.7^{*}$ & $34.3 \pm 20.2$ \\
$\mathrm{LDH} \mathrm{IU} \cdot \mathrm{L}^{-1}$ & $740.3 \pm 808.5^{*}$ & $403.5 \pm 287.6^{*}$ & $372.9 \pm 271.8^{*}$ & $195.5 \pm 81.5$ & $490.6 \pm 333.0^{*}$ & $177.2 \pm 83.1$ \\
WBC $\mu \mathrm{L}$ & $2685 \pm 5153$ & $2487 \pm 2887$ & $1847 \pm 1539$ & $815 \pm 321$ & $2615 \pm 3013$ & $1787 \pm 4871$ \\
Neutrophil \% & $13.75 \pm 10.61$ & $15.45 \pm 7.96$ & $12.78 \pm 8.80$ & $9.07 \pm 6.49$ & $12.30 \pm 8.70$ & $15.00 \pm 10.69$ \\
Lymphocyte \% & $72.87 \pm 13.65^{*}$ & $69.52 \pm 12.60$ & $73.60 \pm 12.73^{*}$ & $72.92 \pm 4.69$ & $70.37 \pm 12.83$ & $68.03 \pm 12.18$ \\
Monocyte \% & $7.05 \pm 6.26^{*}$ & $9.50 \pm 7.61$ & $6.87 \pm 5.08^{*}$ & $12.34 \pm 4.96$ & $9.06 \pm 8.55$ & $9.92 \pm 7.68$ \\
\hline
\end{tabular}

Data are presented as mean \pm SD. CABG: coronary artery bypass grafting; LDH: lactate dehydrogenase; WBC: white blood cell. *: $\mathrm{p}<0.05$ compared with the transudate group. 
This study assesses the usefulness of ADA measurement in lymphocytic effusions. This practice resembles clinical decision making where tuberculosis is most commonly suspected only in lymphocytic effusions. By restricting this study to lymphocytic pleural effusion, false-positives were rare $(<2 \%)$. An elevated pleural fluid ADA level in countries with a high prevalence of tuberculous pleural effusions, as in Spain, has a high degree of specificity for tuberculous pleuritis, which makes it an integral part of the diagnostic work-up of lymphocyte-rich pleural effusions. In areas where the prevalence of disease is low, there is a higher likelihood of false-positive test results, and this can lead to the unnecessary administration of antituberculous therapy or a delay in making an alternative diagnosis such as malignancy. Thus far, high ADA levels in lymphocytic effusions should be looked on as a screening test to guide further diagnostic tests, such as closed pleural biopsy.

In this study, the negative predictive value of the ADA test was very high. The sensitivity and specificity of ADA depends on the prevalence of tuberculosis in the population. With the decline in the prevalence of tuberculous pleural effusion in some areas, the positive predictive value of pleural fluid ADA also declines but the negative predictive value remains high. Therefore, the measurement of the pleural fluid ADA level is an excellent test to rule out a tuberculous aetiology of lymphocytic pleural effusions, irrespective of the rate of prevalence of the disease.

This study is larger than the prospective study of LEE et al. [6], but the results presented by these investigators are similar. The present study included a higher number of malignant and parapneumonic pleural effusions and a lower number of post-CABG effusions, which may more closely resemble the patient population in most pleural units. Moreover, all idiopathic pleural effusions were included in this study, so that the conclusions can be applied to patients with lymphocytic pleural effusions seen in clinical practice.

In view of the rigorous diagnostic criteria for pleural tuberculosis, some cases may have been expected to be missed, particularly when considering that one of the patients with an unknown diagnosis had an elevated ADA. Thus far, the median follow-up among these patients with idiopathic effusions is 21 months and none of them have developed tuberculosis. Nonetheless, it is very unlikely that the current authors' major conclusions would be affected by the diagnostic pitfall, if any, in this group of patients. The present results are in agreement with those of FERRER et al. [19], with $15.6 \%$ of idiopathic effusions in this study. In the study by FERRER et al. [19], none of the patients developed tuberculosis during the follow-up period. In the current study, only one patient with an idiopathic effusion had a high ADA level $\left(63 \mathrm{IU} \cdot \mathrm{L}^{-1}\right)$ but $\mathrm{ADA}_{1} /$ $\mathrm{ADA}_{\mathrm{p}}$ correctly classified this patient as having a nontuberculous effusion. In this particular case, pleural effusion resolved spontaneously without relapses after 47 months of follow-up.

ADA represents the sum of two isoenzymes $\left(\mathrm{ADA}_{1}\right.$ and $\left.\mathrm{ADA}_{2}\right) \cdot \mathrm{ADA}_{1}$ is ubiquitous in all cells, including lymphocytes and monocytes, whereas $\mathrm{ADA}_{2}$ is found only in monocytes. Analysis and determination of these isoenzymes have shown that increases in ADA with tuberculous pleurisy are due to increases in $\mathrm{ADA}_{2}$ and that the $\mathrm{ADA}_{1} / \mathrm{ADA}_{\mathrm{p}}$ ratio improves performance in terms of sensitivity, specificity and efficacy $(100 \%$, $92-97 \%$, and $98 \%$, respectively) in correcting all falsenegative and false-positive results except $1-9 \%$ of nonlymphoproliferative malignancies. The findings of the present study support the use of ADA isoenzymes in cases of suspected nontuberculous lymphocytic pleural effusions with an elevated ADA.

In conclusion, an elevated level of adenosine deaminase activity is seldom found in nontuberculous lymphocytic pleural effusions. An adenosine deaminase level $<40 \mathrm{IU} \cdot \mathrm{L}^{-1}$ virtually excludes tuberculosis in lymphocytic pleural effusions. Adenosine deaminase $_{1} /$ adensine deaminase $_{p}$ ratio improves the performance of adenosine deaminase activity in cases of false-positive findings.

\section{References}

1. Sahn SA. The pleura (state of the art). Am Rev Respir Dis 1988; 138: 184-234.

2. Berger HW, Mejia E. Tuberculous pleurisy. Chest 1973; 63: 88-92.

3. Antony VB, Sahn SA, Antony AC, Repine JE. Bacillus Calmette-Guerin stimulated neutrophiles release chemotaxins for monocytes in rabbit pleural space in vitro. J Clin Invest 1985; 76: 1514-1521.

4. Light RW, Rogers JT, Cheng D, Rodriguez RM. Large pleural effusions occurring after coronary artery bypass grafting. Ann Intern Med 1999; 130: 891-896.

5. Burgess LJ, Maritz FJ, Le Roux I, Taljaard JJ. Combined use of pleural adenosine deaminase with lymphocyte/neutrophil ratio: increased specificity for the diagnosis of tuberculous pleuritis. Chest 1996; 109: $414-419$

6. Lee YC, Rogers JT, Rodriguez RM, Miller KD, Light RW. Adenosine deaminase levels in nontuberculous lymphocytic pleural effusions. Chest 2001; 120 : 356-361.

7. De Oliveira HG, Rossatto ER, Prolla JC. Pleural fluid adenosine deaminase and lymphocyte proportion: clinical usefulness in the diagnosis of tuberculosis. Cytopathology 1994; 5: 27-32.

8. Pérez-Rodríguez E, Pérez-Walton IJ, Sánchez Hernández $\mathrm{JJ}$, et al. $\mathrm{ADA}_{1} / \mathrm{ADA}_{\mathrm{p}}$ ratio in pleural tuberculosis: an excellent diagnostic parameter in pleural fluid. Respir Med 1999; 93: 816-821.

9. Blake J, Berman P. Useful of adenosine deaminase determination for the diagnosis of tuberculosis. $S \mathrm{Afr}$ Med 1982; 62: 782-786.

10. Bañales JL, Pineda PR, Fitzgerald M, Rubio H, Selman M, Salazar-Lezama M. Adenosine deaminase in the diagnosis of tuberculous pleural effusions. A report of 218 patients and review of the literature. Chest 1991; 99: 355-357.

11. Antoniskis D, Amin K, Barnes P. Pleuritis as a manifestation of reactivation tuberculosis. $\mathrm{Am} \mathrm{J} \mathrm{Med}$ 1990; 89: 447-450.

12. Chan C, Arnold M, Mak T. Clinical and pathological features of tuberculous pleural effusion and its longterm consequences. Respiration 1987; 91: 106-109.

13. Ribera E, Martínez Vázquez JM, Ocana I, et al. Gamma interferon and adenosine deaminase in pleuritis. Med Clin 1990; 94: 364-367. 
14. Valdes L, San José E, Alvarez D, et al. Diagnosis of tuberculous pleuresy using the biologic parameters adenosine deaminase, lysozime and interferon gamma. Chest 1993; 103: 458-465.

15. Muranhisi $\mathrm{H}$, Nakashima $\mathrm{M}$, Hirano $\mathrm{H}$, et al. Simultaneous measurements of adenosine deaminase activity and tuberculostearic acid in pleural effusion for the diagnosis of tuberculous pleuritis. Intern Med 1992; 31: 752-755.

16. Ocana I, Ribera E, Martínez Vázquez JM, et al. Adenosine deaminase activity in rheumatoid pleural effusion. Ann Rheum Dis 1988; 47: 394-397.
17. Burgess LJ, Maritz FJ, Le Roux I, Taljaard JJ. Use of adenosine deaminase as a diagnostic tool for tuberculous pleurisy. Thorax 1995; 50: 672-674.

18. Valdés L, Álvarez D, San José E, et al. Value of adenosine deaminase in the diagnosis of tuberculous pleural effusions in young patients in a region of high prevalence of tuberculosis. Thorax 1995; 50: 600-603.

19. Ferrer JS, Muñoz XG, Orriols RM, Light RW, Morell FB. Evolution of idiopathic pleural effusion. A prospective, long-term, follow-up study. Chest 1986; 109: 508-513. 